\title{
Electrospun Gelatin Constructs with Tunable Fiber Orientation Promote Directed Angiogenesis
}

\author{
Ramon B. Montero1, Roberto I. Vazquez-Padron'2, Si M. Pham ${ }^{1,2}$, \\ Gianluca D'Ippolito ${ }^{1,3,4}$, Fotios M. Andreopoulos ${ }^{1,2^{*}}$ \\ ${ }^{1}$ Department of Biomedical Engineering, MCA 219 McArthur Engineering Building, University of Miami, \\ Coral Gables, USA \\ ${ }^{2}$ Daughtry Department of Surgery, Miami Transplant Institute, Highland Professional Building, \\ Miller School of Medicine, Miami, USA \\ ${ }^{3}$ Department of Orthopedics, University of Miami School of Medicine, Miami, USA \\ ${ }^{4}$ Geriatric Research, Education, and Clinical Center and Research Service, Bruce W. Carter, \\ Veterans Affairs Medical Center, Miami, USA \\ Email: ${ }^{\text {fandreopoulos@miami.edu }}$
}

Received 10 January 2014; revised 10 February 2014; accepted 17 February 2014

Copyright (C) 2014 by authors and Scientific Research Publishing Inc.

This work is licensed under the Creative Commons Attribution International License (CC BY). http://creativecommons.org/licenses/by/4.0/

(c) (i) Open Access

\section{Abstract}

The field of therapeutic angiogenesis has been predominantly concentrated in modalities that incorporate pro-angiogenic growth factors and/or cells within polymeric constructs that are implanted into the ischemic region. There is growing evidence that construct architecture can significantly affect growth factor activity, cellular viability and differentiation potential. Electrospinning is an attractive but simple scaffold fabrication technique that offers several advantages over traditional fabrication approaches to prepare highly organized structures for therapeutic angiogenesis applications. We recently described the fabrication of nanofibrous scaffolds with aligned fiber orientation that directed cell migration and orientation (i.e. human umbilical vein endothelial cells). Herein we demonstrate the ability of bFGF containing nanofibrous gelatin B scaffolds with controlled fiber orientation to promote capillary formation in vivo. Aligned scaffolds loaded with bFGF induced the highest levels of reperfusion (73\% increased in LDPI ratios by day 21 post ischemia induction) in comparison to all other groups including scaffolds with random fiber orientation. Furthermore, the newly formed vasculature, assessed by confocal microscopy, had a parallel alignment along the axis of the scaffold's fibers. In contrast, no vessel directionality was observed in the animals treated with scaffolds with random fiber orientation in the presence or absence of bFGF.

*Corresponding author.

How to cite this paper: Montero, R.B., Vazquez-Padron, R.I., Pham, S.M., D'Ippolito, G. and Andreopoulos, F.M. (2014) Electrospun Gelatin Constructs with Tunable Fiber Orientation Promote Directed Angiogenesis. Open Journal of Regenerative Medicine, 3, 1-12. http://dx.doi.org/10.4236/ojrm.2014.31001 


\section{Keywords}

\section{Electrospinning; bFGF; Directed Angiogenesis; Vessel Formation; Critical Limb Ischemia; Scaffold Fabrication}

\section{Introduction}

Diseases that result in ischemia due to vascular occlusions are the leading causes of morbidity and mortality in western societies despite improvements in minimally invasive surgical interventions to reestablish proper blood flow in underperfused regions of the heart and legs. Current therapeutic angiogenesis strategies to revascularize the area of ischemia involve the administration (e.g. intravenous or intramuscular) of angiogenic growth factors (e.g. VEGF, bFGF, Angiopoetin 1), the transplantation of cells (e.g. bone marrow mononuclear cells, mesenchymal stem cell or endothelial progenitor cells) or a combination of cell and cytokine therapy [1]-[4]. Tissue engineering approaches also include the use of biomaterials and scaffolds for the localized delivery and/or protection of the administered molecules and cells. A critical aspect of these investigative strategies involves the spatiotemporal control of vasculogenesis since undirected vessel growth and disorganized vascular patterning can lead to immature vessel formation and poor network functionality. Even though a number of tissue engineering approaches to promoting revascularization have been reported, minimal success has been achieved in controlling the direction and organization of the newly formed vasculature [5]-[7].

Angiogenic processes are primarily dictated by the orchestrated interactions among cytokines, cells and the extra cellular matrix (ECM) [8]. During embryogenesis as well as in adult tissue repair processes, the ECM dictates the function and phenotype of various cells [9]-[11]. In an attempt to create scaffolds that closely mimic the biological, chemical and structural characteristics of the ECM, novel scaffold fabrication techniques have been developed for tissue engineering applications. Recently, electrospinning has emerged as a viable and popular method for scaffold fabrication since it allows the production of nanoscale fibers that closely resemble the natural ECM in both structure and scale. The electrospinning process is comprised of a polymeric solution drawn from a needle tip by an electrostatic force generated from a high voltage power supply. The manipulation of fiber deposition and orientation is of particular interest in all areas of tissue engineering where control of cell morphology, migration and orientation is desired. In therapeutic angiogenesis, several groups have attempted to design drug-loaded and architecturally relevant nanofibrous scaffolds in order to promote cellular behavior and mimicry of the native vascular tissue [12]-[16]. For example, Ma et al. documented the orientation of endothelial cells (ECs) to be parallel to the direction of the nanofiber alignment, in contrast to the random cellular orientation seen in randomly deposited nanofibrous scaffolds [3]. Similar results have also been reproduced in our laboratory using human umbilical vein endothelial cells (HUVECs) [17]. Qian et al. demonstrated that endothelial cells seeded onto aligned nanofibrous scaffolds in contrast to those seeded onto randomly deposited nanofibrous scaffolds, preserved the expression of platelet-endothelial cell adhesion molecule 1 (PECAM-1), intercellular adhesion molecule 1 (ICAM-1) and vascular cell adhesion molecule 1 (VCAM-1) [18]. Recently, Kumar et al. reported the effects of different scaffold architectures on primary human bone marrow stromal cells (hBMSCs) [19]. They demonstrated the ability of scaffold architecture and structure to dictate cell morphology and consequently induce unique gene expressions. The aligned electrospun scaffold promoted the differentiation of hBMSCs to osteogenic cells in the absence of osteogenic supplements [19]. Similar results have been reported for smooth muscle cells (SMCs) [20] and human aortic endothelial cells (HAECs) [21] in which scaffold architecture was able to induce desired cellular morphology and consequently preserve desired cell markers. Zhang et al. successfully cultured human aortic endothelial cells (HAECs) upon silk fibroin scaffolds [21]. The cells, following seven days of culturing, maintained phenotypic cell markers (i.e. CD146, VE-Cadherin, PECAM-1 and vWF) and formed short cord-like structures that began to interconnect and form a network of capillaries with identifiable lumens [21].

We have developed a novel approach to fabricating gelatin B electrospun scaffolds with tunable fiber orientation to direct endothelial cell migration and consequently dictate the pattern of the newly formed vasculature, via electrostatic field manipulation. In a recent publication, we demonstrated that HUVEC morphology and capillary sprouting are influenced by the architectural features of the electrospun scaffolds (i.e. fiber dimension and 
orientation) in an in-vitro setting [17]. Herein we proceed to assess the angiogenic potential of these constructs on an ischemic mouse hind-limb model (in-vivo) and their ability to promote and direct vascular growth.

\section{Materials and Methods}

\subsection{Scaffold Fabrication, Crosslinking and bFGF Loading}

Gelatin B (Sigma Aldrich USA, Gelatin Type B from Bovine Skin G9391-500G) was dissolved at 10\% w/v in 1,1,1,3,3,3 hexafluoro-2-propanol (Sigma Aldrich USA, Fluka HFIP 52512-100 mL), the solution was loaded into a $5 \mathrm{~mL}$ syringe (Thomas Scientific USA, Henke Sass Wolf Norm-Ject 8939N52) of our electrospinning apparatus and scaffolds were fabricated according to conditions described in our previous publication [17]. Briefly, the $10 \%$ gelatin B solution in HFIP was electrospun at $15 \mathrm{kV}, 10 \mathrm{~cm}$ needle-to-collector distance, $4 \mathrm{~mL} / \mathrm{hr}$ feedrate, and a total volume of $500 \mu \mathrm{L}$ deposited onto an aluminum covered glass slide and left to dry for 1 hour under vacuum (25 in Hg) at room temperature. Aligned fiber orientation was achieved by controlling the polarity of the collecting plates of our custom made electrospinning apparatus [17]. Crosslinking of gelatin B nanofibrous scaffolds was performed with glutaraldehyde vapor (Sigma Aldrich USA, Glutaraldehyde Solution Grade II $25 \%$ in $\mathrm{H}_{2} \mathrm{O}$ G6257-1L). Briefly, $2 \mathrm{~mL}$ of $25 \%$ glutaraldehyde solution per scaffold was deposited into a standard container which in turn was placed alongside the scaffold into an airtight container. Scaffolds were crosslinked for one hour at room temperature and then washed for 1 hour in $100 \mathrm{mM}$ glycine solution (Sigma Aldrich) to block any unreacted aldehyde groups. Once the wash is finalized the rectangular scaffold was cut into $10 \mathrm{~mm}$ disks using a $10 \mathrm{~mm}$ disposable punch biopsy. Scaffolds were then stored in PBS pH 7.4 until ready for use. Prior to implantation, scaffolds were washed with $70 \%$ alcohol for 30 minutes and rinsed three times with PBS for 30 minutes. Once scaffolds were washed and rinsed, they were air dried under a biological hood for 15 minutes. Immediately prior to implantation a $10 \mu \mathrm{L}$ aliquot containing $1000 \mathrm{ng}$ of bFGF (human recombinant, Cat. No. PHG0021, MW = 17 kDa Gibco,) was pipetted in the center of the $10 \mathrm{~mm}$ disk shaped scaffold and allowed to absorb.

\subsection{Angiogenesis Assessment in a Critical Hind Limb Ischemic Model}

The murine critical limb ischemic model mimics several aspects of human occlusive artery disease and has been extensively used to investigate vascular regeneration in the presence of various therapeutic modalities. The mouse hind limb is perfused by the common femoral artery which after passing the inguinal ligament gives rise to the deep femoral branch, the A. profunda femoris. This branch divides into collateral vessels that perfuse the semimembranosus and semitendinosus muscle of the limb and therefore ligation of the femoral artery induces ischemia distally to the gastrocnemius muscle of the calf [22].

We hypothesized that aligned fibrous scaffolds loaded with bFGF would have an advantage over their randomly deposited counterparts in directing vessel growth and thus achieving faster reperfusion of the ischemic tissue. Bolus or systemic administration of angiogenic factors from a delivery vehicle promotes new capillary formation however the newly formed vasculature is chaotic and usually concentrated around the GF's delivery sight [23]. In order to assess the angiogenic response of the bFGF-containing microfibrous scaffolds as a function of fiber architecture, the following groups were evaluated (Figure 1):

1) bFGF bolus administration-no scaffold

2) Electrospun gelatin B scaffolds with random fiber orientation loaded with bFGF

3) Electrospun gelatin B scaffolds with aligned fiber orientation loaded with bFGF

4) Electrospun gelatin B scaffolds with random fiber orientation-No bFGF

5) Electrospun gelatin B scaffolds with aligned fiber orientation-No bFGF

6) Saline

Critical ischemia was induced in Balb/C mice, 6 - 7 weeks of age (weight of 30 - 40 grams) by ligating the deep femoral artery below the neurovascular bundle and above the collateral arteries (Figure 2). The animal procedures were approved by the Institutional Animal Care and Use Committee at the University of Miami according to the guidelines set by The Guide For the Care and Use of Laboratory Animals. The mice were anesthetized with an intraperitoneal injection of ketamine $(100 \mathrm{mg} / \mathrm{mL})$ at a dose of $80-100 \mathrm{mg} / \mathrm{kg}$ and xylazine (20 $\mathrm{mg} / \mathrm{mL}$ ) at a dose of $4-6 \mathrm{mg} / \mathrm{kg}$ [2]. Once anesthetized, the mouse was placed on a $37^{\circ} \mathrm{C}$ heating pad and benpanthen ophthalmic ointment was applied to the eyes. Following, the lower abdominal and groin area were 


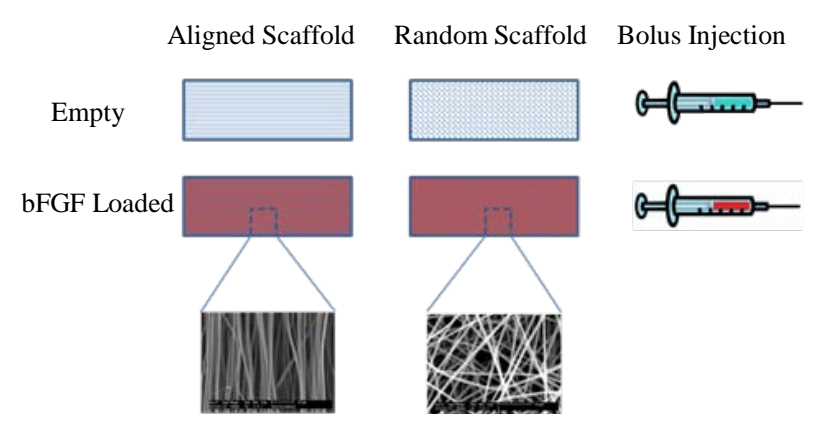

Figure 1. Schematic representation of treatment groups to be administered on the ischemic region of the murine hindlimb.
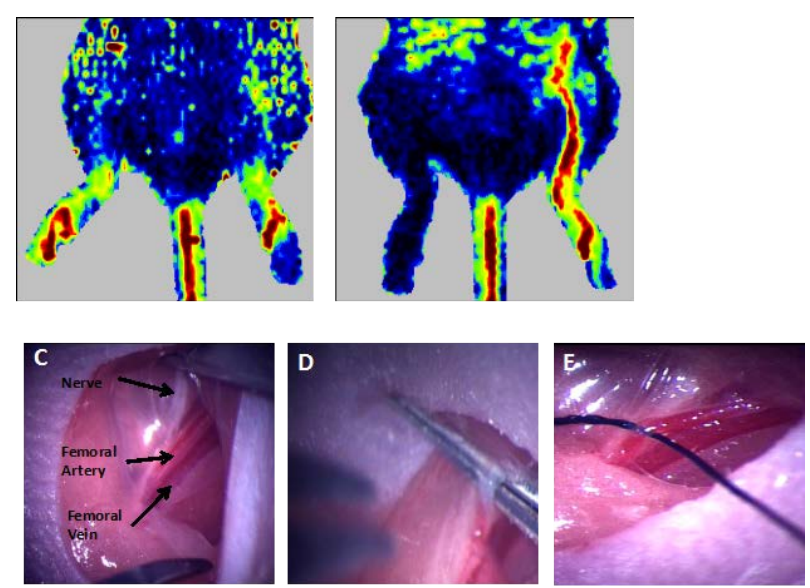

Figure 2. Representation of critical limb ischemia induction in a murine model. (A) LDPI image prior to surgical procedure demonstrating blood flow in both hind limbs. (B) LDPI image immediately after surgery demonstrating loss of blood flow on the right hind limb. (C) Initial incision on the inguinal crease showing the neurofemoral bundle landmark. (D) Separation of femoral artery from femoral vein and nerve using Miltex \#5 Swiss micro-forceps. (E) Threading of femoral artery with 6-0 silk braided suture.

shaved and Veet or Nair hair removal cream was applied to remove all remaining hair. The area was then cleaned with warm PBS and disinfected with povidone iodine pads. A $5 \mathrm{~mm}$ longitudinal incision beginning at the inguinal crease along the femoral vessels visible through the skin was made using a Miltex iris scissors 4" and Miltex \#5 swiss micro-forceps, both from Integra LifeSciences (Miltex York, PA). Once the anatomical landmarks were identified the femoral artery was ligated above and immediately below the deep femoral branch using triple surgical knots with 6-0 Ethicon Perma-Hand Silk black braided sutures in order to block perfusion via collateral arteries and thus cause critical ischemia. The ligated arterial region was then excised. All treatments were administered prior to skin closure by first detaching the skin by gently sliding the Miltex iris scissors under the incision and applying upward pressure to break the interstitial tissue. The gelatin B scaffolds were carefully tucked under the skin covering the ligation point under the femoral branch and extending all the way down towards the knee. (Figure 3) After implantation the incision was closed using triple surgical knots with a 5-0 Ethicon Silk suture.

\subsection{Experimental Set-Up and Statistical Analysis}

In order to assess significance of treatment modalities on their ability to induce and direct angiogenesis we tested 5 mice per treatment group for a total of 30 mice. All five mice per group where assessed using Laser Doppler Perfusion Imaging on days 5, 7, 10, 14, and 21, as well as pre and post-surgery for baseline purposes. 
(a)

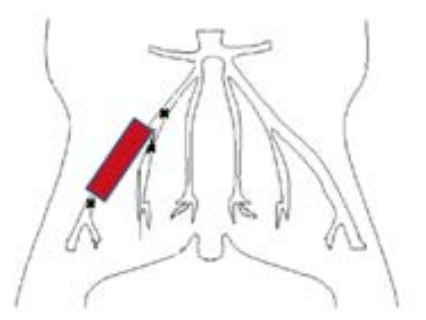

(b)

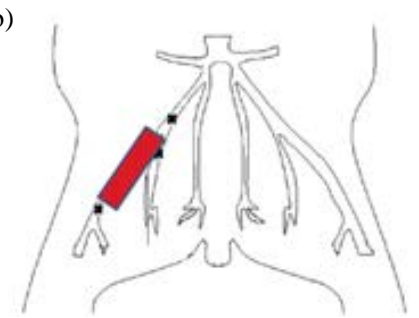

(c)

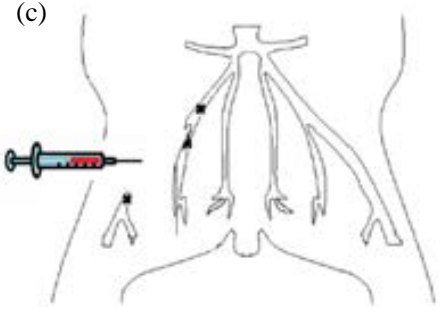

Figure 3. Schematic representation of the placement of the treatment groups. (A) Aligned gelatin B scaffold treatment with or without bFGF, (B) random gelatin B scaffold treatment with or without bFGF, and (C) bolus injection of either bFGF or saline solution.

On day 21, three (3) mice per treatment were sacrificed for vessel bed imaging via Lipophilic Tracer DiI, and the remaining two were used for the immunohistochemistry studies.

Statistical analysis was performed on the LDPI images that were collected from all animals per treatment group. Two CD31 stained immunohistochemistry slides per animal per group were evaluated to determine the number of formed vessels per unit area and vessel diameter. Two images per slide were taken covering an area of $1.75 \mathrm{~mm} \times 1.25 \mathrm{~mm}$ each, in which all the vessels were counted, measured, and averaged for statistical analysis. The statistical method used was the standard t-test function in the Excel software in which two sets of unequal variance data (heteroscedastic data) are evaluated under a two-tailed distribution. All statistically relevant data (at $\mathrm{p}<0.05$ ) in the figures is depicted by an asterisk $(*)$.

\subsection{Angiogenic Assessment}

\subsubsection{Laser Doppler Perfusion Imaging}

Blood perfusion in the ischemic hind limbs was measured using Laser Doppler Perfusion Imaging analysis (LDPI; Perimed, New York, USA). LDPI uses a low power He-Ne laser to scan living animals and is therefore suitable for serial studies. Blood cells in motion give rise to Doppler shifts in the reflected light, which were recorded and converted into perfusion values, as the magnitude and frequency distribution of the Dopplershifted light are directly related to the number and velocity of blood cells. The Doppler signal is linearly proportional to perfusion of the upper $200-300 \mu \mathrm{m}$ of the skin [24]. Tissue perfusion is quantified comparatively in the limbs relative to the contralateral, non-ligated side, and can be displayed as color-coded images [25]. The feet were scanned prior to surgery, immediately post-surgery, 5, 7, 10, 14, and 21 days after surgery. A Doppler ratio was determined by a comparison of the average Doppler intensity at the time of the scan versus the baseline scan prior to surgery of the right hindlimb.

\subsubsection{Vessel Bed Imaging via Lypophilic Tracer}

Vessel formation and branching was determined 3 weeks post-surgery using vessel painting with a lipophilic tracer, carbocyanine dye DiI [2]. Based on previous methods [26], mice were euthanized via $\mathrm{CO}_{2}$ overdose and a transverse incision made on the abdominal area to open the abdominal cavity. The exposed diaphragm was then cut as well as the chest wall on both sides up to the third rib. Once the heart was exposed, a 25GA butterfly needle was inserted into the left ventricle followed by puncturing the right atrium. The vasculature was flushed with $10 \mathrm{ml}$ of PBS injected through the right atrium at a rate of $1-2 \mathrm{ml} / \mathrm{min}$. After the blood was flushed out, 10 $\mathrm{ml}$ of DiI working solution was injected at a rate of $1-2 \mathrm{ml} / \mathrm{min}$. No additional dye was needed once the digits turned a light red/pink color. Finally, $10 \mathrm{~mL}$ of $4 \%$ paraformaldehyde was injected at a rate of $1-2 \mathrm{~mL} / \mathrm{hr}$ in order to fix the tissue. After the procedure was completed, the entire hind limb was removed and preserved in $4 \%$ paraformaldehyde solution for confocal imaging. Images were taken on a Leica SP-5 inverted confocal microscope with a $5 \mathrm{X}$ lense and a rhodamine filter [2].

\subsubsection{Immunohistochemistry}

Following animal euthanasia the hind limb muscle was removed and tissue samples preserved in $10 \%$ formaldehyde were shipped to American HistoLabs Inc. for CD31+ staining. Stained slides were examined for CD31 positive cells/vessels in the area of scaffold implantation, directly on top of the deep femoral branch expanding over the semimembranosus and semitendinosus muscles. Positively stained CD31 capillaries were manually 
counted in minimum five separate fields and expressed as capillaries per millimeter squared. Slides were viewed using light microscopy at 60X using a LEICA Microsystems AF6000LX brightfield/fluorescence microscope (Bannockburn, IL), and images were captured using MetaMorph 5.1 Software. Images taken from the fluorescent microscope captured an area of $1.75 \mathrm{~mm} \times 1.25 \mathrm{~mm}$.

\section{Results and Discussion}

\subsection{Angiogenesis Assessment of bFGF Loaded Electrospun Gelatin B Scaffolds}

\subsubsection{Laser Doppler Perfusion Imaging}

The effects of bFGF and scaffold architecture in reestablishing blood flow to underperfused tissue were evaluated in vivo with Laser Doppler Perfusion Imaging (LDPI).

The images obtained from the Perimed Laser Perfusion Imaging device where analyzed using the LDPIwin program included with the apparatus (Figure 4(A)). The Doppler intensities were normalized for both pre- and post-surgery images and used as baselines for the rest of the measurements to calculate the percent perfusion restoration (Figure 4(B)). Aligned scaffolds loaded with bFGF demonstrated the most significant $(\mathrm{p}<0.05)$ reperfusion effect compared to the rest of the treatment modalities on day 14 and day 21 with $73.1 \% \pm 1.8 \%$ and $72.1 \% \pm 3.1 \%$ restoration of the original pre-surgery blood flow, respectively. Scaffolds (random or aligned) without the presence of bFGF also promoted significantly higher levels of reperfusion (increase of about 50\%) in comparison to untreated groups at day 21, by providing a surface for new capillaries to form during the initial inflammatory response.

During the early points of treatment (day 5), scaffolds that were not loaded with bFGF demonstrated low levels of reperfusion (about 7-fold lower) at the site of ligation (white box area, Figure 5(D)) in comparison to the groups that contain bFGF. By day 7 the difference on the levels of reperfusion at the ligation site was much less pronounced among the treatment groups. (Figure 5(D)) However, reperfusion measured at foot (Figure 5(C)) at day 7 yielded significantly better results for the aligned scaffolds loaded with bFGF ( $43.8 \% \pm 3.3 \%$ ) in comparison to all other groups. It's conceivable that the aligned fiber orientation of the implanted scaffolds guided the formation of new vessels along the axis of the fibers from the ligation point down to the foot. The scaffold's architecture appears to have a pronounced effect on directing vessel growth while the addition of bFGF further accelerates angiogenesis.

\subsubsection{Lipophilic Tracer Direct Vessel Bed Imaging}

In order to evaluate the directionality of the newly formed capillaries as a function of scaffold architecture a direct vessel labeling technique utilizing DiI was performed on mice on day 21. Laser confocal microscopy was used to scan the entire scaffold area and ligation point in order to visualize the newly formed vessels. The images encompass an area of $3.10 \mathrm{~mm}^{2}$ and go to a depth of approximately $1.1 \mathrm{~mm}$ with image sections taken in 10 $\mu \mathrm{m}$ steps. The compiled images demonstrate a profound effect on vessel architecture as a direct result of nanofiber alignment (Figure 6). The vessel beds of mice treated with aligned scaffolds with bFGF yielded long straight vessels parallel to the nanofiber alignment in the scaffold (Figure 6(A)). In contrast, vessels that grew on random scaffolds show more spotted vessel architecture rather than well-formed tubes (Figure 6(B)). In the absence of scaffolds the administration of bFGF led to a random and lower density capillary formation (Figure 6(C)). Similar vessel orientation was observed in the animals that were treated with scaffolds in the absence of the immobilized bFGF but at much lower densities. The number of newly formed capillaries was significantly higher in the groups that were treated with the aligned scaffolds that were loaded with bFGF. Specifically, the vessel density (number of vessels per unit area) in that group was almost seven fold higher (33 vessels $/ \mathrm{mm}^{2}$ ) than all other groups (average around 5 vessels $/ \mathrm{mm}^{2}$ ) indicating the significant effect that scaffold topography has on vessel formation. There were no statistical differences on vessel diameter between the scaffold treatment groups; the vessel diameters ranged between $6 \mu \mathrm{m}$ to $30 \mu \mathrm{m}$ (Figure 7(B)).

\subsubsection{Immunohistochemistry}

To corroborate the confocal microscopy data, tissue samples around the area of scaffold implantation were collected at specified time points and stained for a CD31 marker to assess the presence of newly formed capillaries. CD31 positive vessels and cells were visualized under a fluorescent microscope through a Texas Red filter. The animals that were treated with aligned scaffolds loaded with bFGF had the highest amount of newly formed 

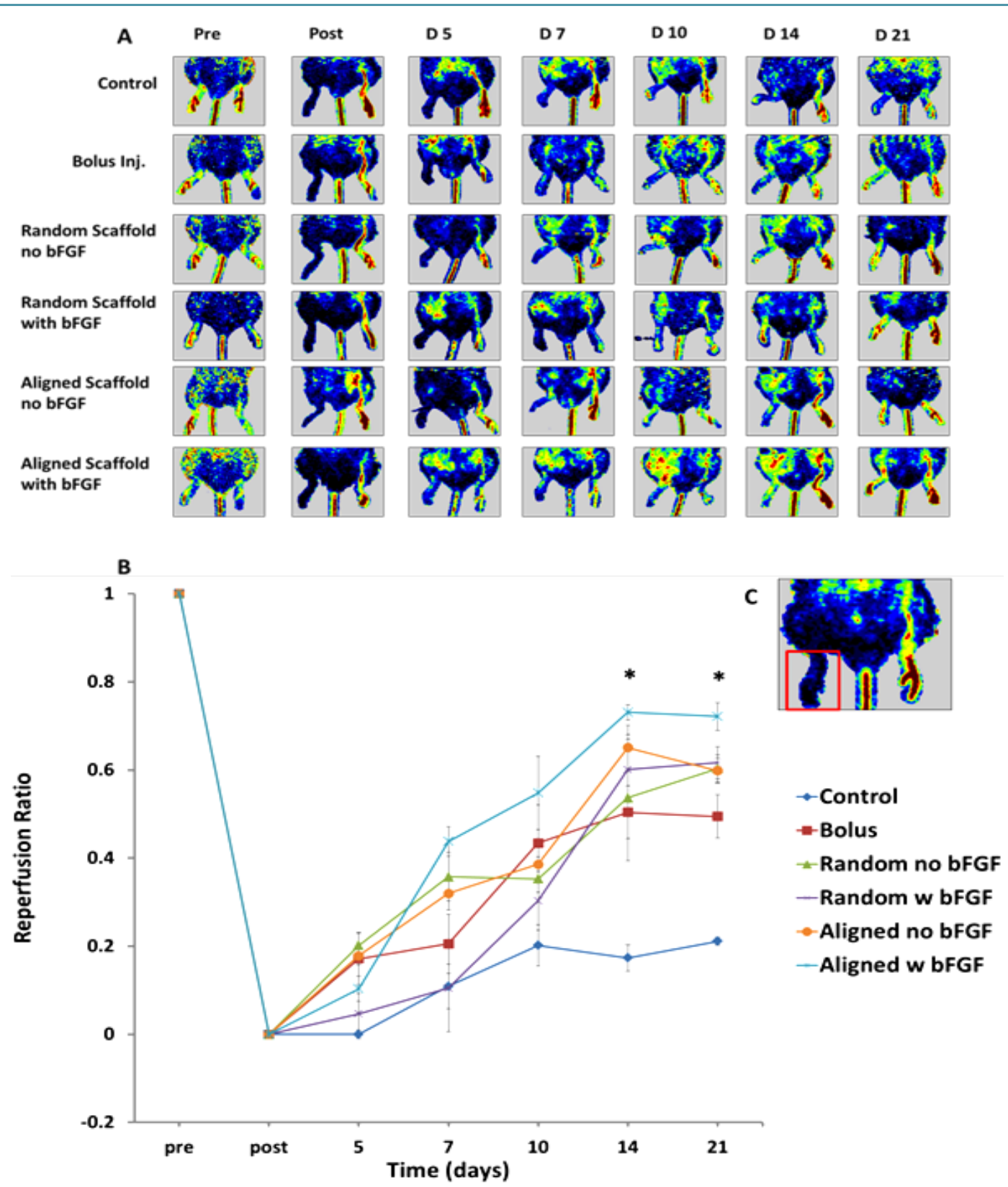

Figure 4. Blood perfusion of the ischemic hindlimb as a function of construct design over 21 days following implantation in the mouse's hindlimb. (A) Representative LDPI images taken with the Perimed Laser Doppler Perfusion Imaging device at pre and post-surgery, and days 5, 7, 10, 14, and 21. (B) Reperfusion of the hindlimb as a function of treatment groups over three weeks post treatment. Percent reperfusion values were calculated from five mice images, as represented by A, per time point. (C) Sample LDPI image depicting region of interest (enclosed in box). Statistical significance was determined when $\mathrm{p}<0.05$ as calculated by a two-tailed standard t-test of heteroscedastic data $(n=5)$, and is denoted by an asterisk $(*)$ between treatments. A total of 30 mice were assessed; 5 animals per treatment group were used.

vessels (33 vessels $/ \mathrm{mm}^{2}$ ) in comparison to all other groups (Figures 7(A) and 8). Furthermore new vessels were found directly on top of the deep femoral branch extending from the semimembranosus to the semitendinosus muscle and appear to have a parallel orientation along the axis of the scaffold fibers that is in agreement with our DiI vessel painting evaluation (Figure 8(A)).

On the contrary, in the case of scaffold with random orientation or bolus treatment fewer vessels were formed and pockets of high concentration of CD31 positive cells were found throughout the gastrocnemius muscle 
A

Control

D 5

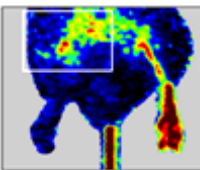

D 7

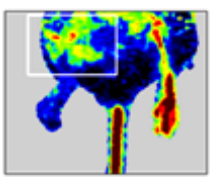

Bolus Inj.
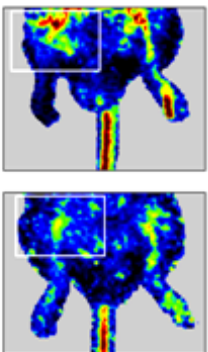

Random Scaffold no bFGF
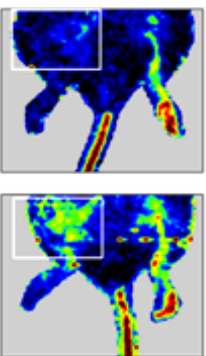

Random Scaffold with bFGF
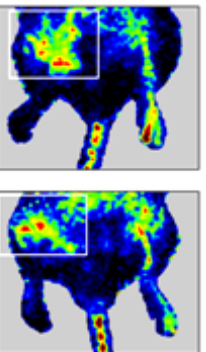

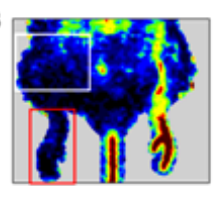

Aligned Scaffold no bFGF
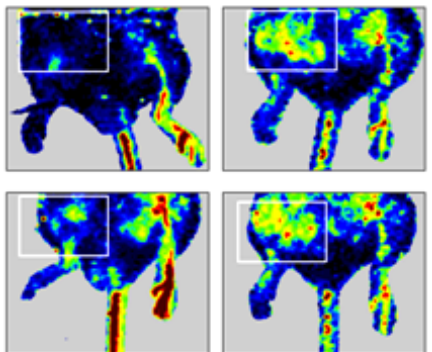
with bFGF

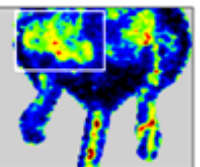

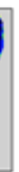
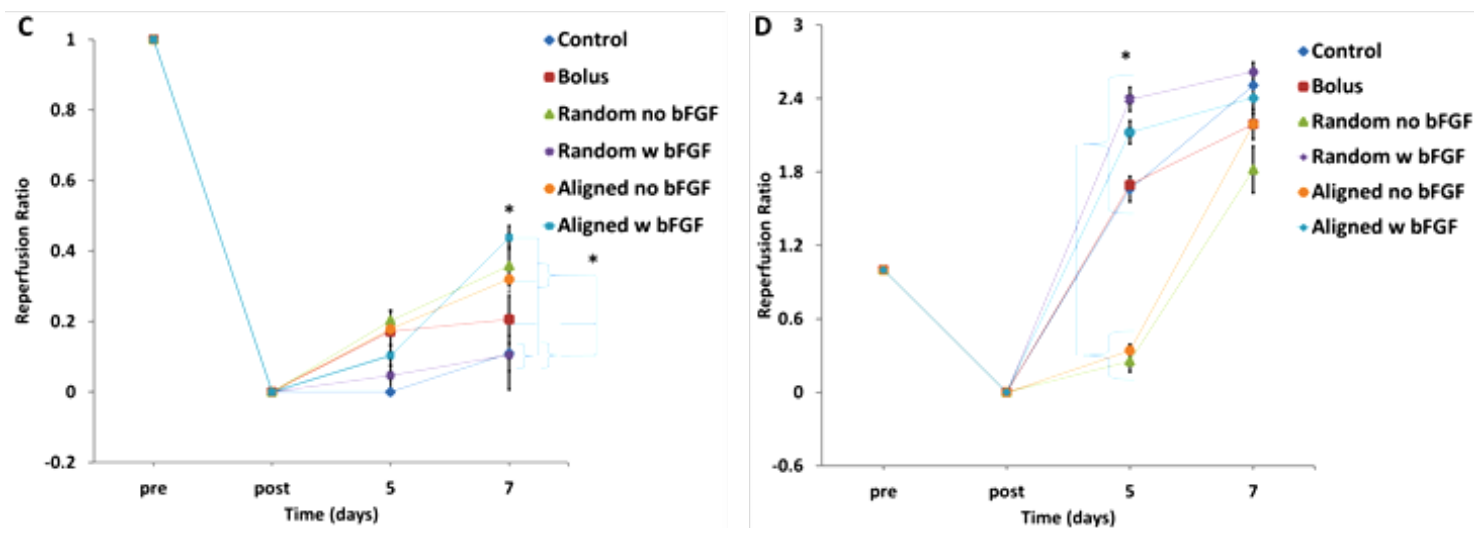

Figure 5. LDPI images from all groups between days 5 and 7 with reperfusion graphs of foot (C) and ligation area (D). (A) LDPI images of the ischemic legs as a function of variable treatment groups over 21 days. Ligation areas are encompassed by a white quadrangle. (B) Sample LDPI image depicting region of interest (ROI, enclosed by red box) on the foot of the mouse. (C) LDPI ratios at the foot of the mouse over 7 days. (D) LPDI ratios at the ligation point over 7 days. Statistical significance was determined when $\mathrm{p}<0.05$ as calculated by a two-tailed standard t-test of heteroscedastic data $(\mathrm{n}=5)$, and is denoted by an asterisk $(*)$ between treatments. A total of 30 mice were assessed.
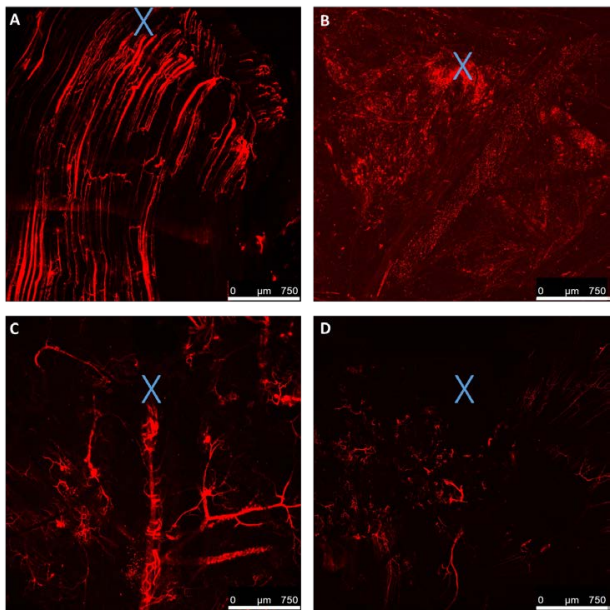

Figure 6. The hind limbs of Balb/C mice were perfused with DiI stain and imaged under a confocal microscope 3 weeks after surgery. Images depict vessel beds at ligation point (marked by $\mathrm{X}$ ) below the neurofemoral bundle showing an area of $3.10 \mathrm{~mm} \times 3.10 \mathrm{~mm}$ and a depth of $0.8 \mathrm{~mm}-1.1 \mathrm{~mm}$. (A) Aligned scaffold with bFGF, (B) random scaffold with bFGF, (C) bolus injection with bFGF, (D) control saline treatment. 

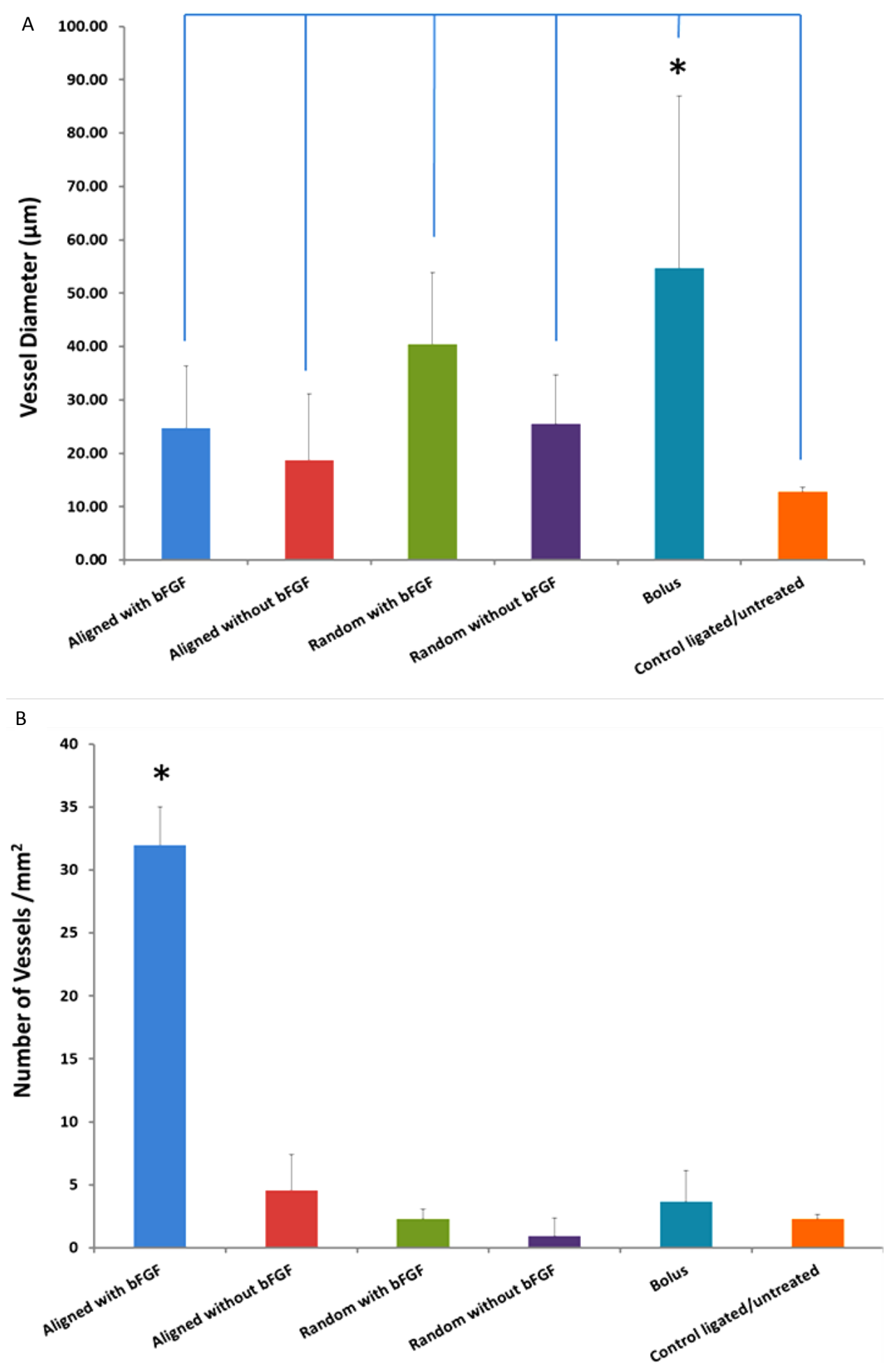

Figure 7. (A) Vessel diameters based on CD31 immunohistochemistry as a function of treatment groups. The examined area was directly above the scaffold implantation site between the semimembranosus and semitendinosus muscles covering a cross-sectional area of $1.75 \mathrm{~mm} \times 1.25 \mathrm{~mm}$. (B) Number of newly formed vessels per unit area with respect to variable treatment groups at 21 days post ischemia. Statistical significance at $\mathrm{p}<0.05$ was assigned by an asterisk $(*)$ and determined via a standard two-tailed t-test of data with different variance. A total of two slides per group and two images per slide were assessed. 

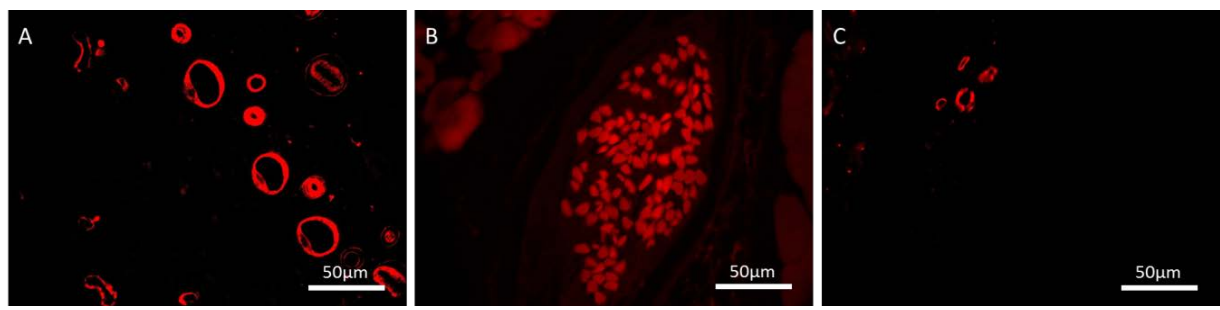

Figure 8. High magnification images depicting vessel formation in (A) scaffolds with aligned fiber orientation loaded with bFGF, (B) non bFGF containing groups, and (C) non-ligated/untreated control. Groups lacking bFGF had numerous pockets of CD31+ cells around the muscle tissue.

(Figure 8(B)). Vessel diameter measurements based on the CD31 vessel staining ranged from 10 to 50 micrometers in agreement with the vessel painting assessment (Figure 8(A)). These results are in accordance to vessel diameters measured by other researchers, who reported diameter means between 22 and $30 \mu \mathrm{m}$ [27] [28].

\section{Conclusion}

In this study, we examined the effects of bFGF loading and scaffold nano-architectural queues on vessel formation and direction in a murine hind limb ischemic model. Scaffold fabrication was achieved by utilizing a novel electrospinning approach capable of producing nanofibrous constructs with patterned fiber architecture which had previously been reported to affect cellular morphology [17]. Scaffold fiber orientation had a pronounced effect of vessel formation and direction. Aligned scaffolds loaded with bFGF promoted new vessel formation from the ligation point down to the foot. The newly formed vessels had a parallel orientation along the axis of the nanofibers, unequivocally demonstrating the effect of construct architecture on vessel patterning. No vessel alignment was observed for constructs with random fiber orientation or in the case of bolus bFGF treatment. We are currently investigating the significance of vessel orientation on tissue preservation and limb function.

\section{Acknowledgements}

The authors would like to acknowledge the support of the National Institutes of Health (NIH-NIBIB R21EB012136 to FMA) for the completion of this work.

\section{Financial Disclosure Statement}

The authors have no financial relationship with any of the companies listed in this manuscript and have nothing to disclose.

\section{References}

[1] Layman, H., Sacasa, M., Murphy, A.E., Murphy, A.M., Pham, S.M. and Andreopoulos, F.M. (2009) Co-Delivery of FGF-2 and G-CSF from Gelatin-Based Hydrogels as Angiogenic Therapy in a Murine Critical Limb Ischemic Model. Acta Biomater, 5, 230-239. http://dx.doi.org/10.1016/j.actbio.2008.07.024

[2] Layman, H., Rahnemai-Azar, A.A., Pham, S.M., Tsechpenakis, G. and Andreopoulos, F.M. (2011) Synergistic Angiogenic Effect of Codelivering Fibroblast Growth Factor 2 and Granulocyte-Colony Stimulating Factor from Fibrin Scaffolds and Bone Marrow Transplantation in Critical Limb Ischemia. Tissue Engineering, Part A, 17, $243-254$. http://dx.doi.org/10.1089/ten.tea.2010.0270

[3] Ma, Z., He, W., Yong, T. and Ramakrishna, S. (2005) Grafting of Gelatin on Electrospun Poly(caprolactone) Nanofibers to Improve Endothelial Cell Spreading and Proliferation and to Control Cell Orientation. Tissue Engineering, 11, 1149-1158. http://dx.doi.org/10.1089/ten.2005.11.1149

[4] Zisch, A.H., Lutolf, M.P. and Hubbell, J.A. (2003) Biopolymeric Delivery Matrices for Angiogenic Growth Factors. Cardiovascular Pathology, 12, 295-310. http://dx.doi.org/10.1016/S1054-8807(03)00089-9

[5] Bouta, E.M., McCarthy, C.W., Keim, A., Wang, H.B., Gilbert, R.J. and Goldman, J. (2011) Biomaterial Guides for Lymphatic Endothelial Cell Alignment and Migration. Acta Biomater, 7, 1104-1113.

http://dx.doi.org/10.1016/j.actbio.2010.10.016

[6] Hadjizadeh, A. and Doillon, C.J. (2010) Directional Migration of Endothelial Cells towards Angiogenesis Using Po- 
lymer Fibres in a 3D Co-Culture System. Journal of Tissue Engineering and Regenerative Medicine, 4, 524-531. http://dx.doi.org/10.1002/term.269.

[7] Heath, D.E., Lannutti, J.J. and Cooper, S.L. (2010) Electrospun Scaffold Topography Affects Endothelial Cell Proliferation, Metabolic Activity, and Morphology. Journal of Biomedical Materials Research Part A, 94A, 1195-1204.

[8] Spadaccio, C., Chello, M., Trombetta, M., Rainer, A., Toyoda, Y. and Genovese, J.A. (2009) Drug Releasing Systems in Cardiovascular Tissue Engineering. Journal of Cellular and Molecular Medicine, 13, 422-439. http://dx.doi.org/10.1111/j.1582-4934.2008.00532.x

[9] Eble, J.A. and Niland, S. (2009) The Extracellular Matrix of Blood Vessels. Current Pharmaceutical Design, 15, 13851400. http://dx.doi.org/10.2174/138161209787846757

[10] Chien, K.R., Domian, I.J. and Parker, K.K. (2008) Cardiogenesis and the Complex Biology of Regenerative Cardiovascular Medicine. Science (Washington DC, U S), 322, 1494-1497. http://dx.doi.org/10.1126/science.1163267

[11] Lutolf, M.P. and Hubbell, J.A. (2005) Synthetic Biomaterials as Instructive Extracellular Microenvironments for Morphogenesis in Tissue Engineering. Nature Biotechnology, 23, 47-55. http://dx.doi.org/10.1038/nbt1055

[12] Bu, X., Yan, Y., Zhang, Z., et al. (2010) Properties of Extracellular Matrix-Like Scaffolds for the Growth and Differentiation of Endothelial Progenitor Cells. Journal of Surgical Research, 164, 50-57. http://dx.doi.org/10.1016/j.jss.2009.03.018

[13] Hu, X., Shen, H., Yang, F., Bei, J. and Wang, S. (2008) Preparation and Cell Affinity of Microtubular OrientationStructured PLGA(70/30) Blood Vessel Scaffold. Biomaterials, 29, 3128-3136. http://dx.doi.org/10.1016/j.biomaterials.2008.04.010

[14] Indolfi, L., Baker, A.B. and Edelman, E.R. (2012) The Role of Scaffold Microarchitecture in Engineering Endothelial Cell Immunomodulation. Biomaterials, 33, 7019-7027. http://dx.doi.org/10.1016/j.biomaterials.2012.06.052

[15] Moroni, L., Licht, R., de Boer, J., de Wijn, J.R. and van Blitterswijk, C.A. (2006) Fiber Diameter and Texture of Electrospun PEOT/PBT Scaffolds Influence Human Mesenchymal Stem Cell Proliferation and Morphology, and the Release of Incorporated Compounds. Biomaterials, 27, 4911-4922. http://dx.doi.org/10.1016/j.biomaterials.2006.05.027

[16] Ragetly, G.R., Griffon, D.J., Lee, H.-B., Fredericks, L.P., Gordon-Evans, W. and Chung, Y.S. (2010) Effect of Chitosan Scaffold Microstructure on Mesenchymal Stem Cell Chondrogenesis. Acta Biomaterialia, 6, 1430-1436. http://dx.doi.org/10.1016/j.actbio.2009.10.040

[17] Montero, R.B., Vial, X., Nguyen, D.T., et al. (2012) bFGF-Containing Electrospun Gelatin Scaffolds with Controlled Nano-Architectural Features for Directed Angiogenesis. Acta Biomaterialia, 8, 1778-1791. http://dx.doi.org/10.1016/j.actbio.2011.12.008

[18] Qian, T. and Wang, Y. (2010) Micro/Nano-Fabrication Technologies for Cell Biology. Medical \& Biological Engineering \& Computing, 48, 1023-1032. http://dx.doi.org/10.1007/s11517-010-0632-z

[19] Kumar, G., Tison, C.K., Chatterjee, K., et al. (2011) The Determination of Stem Cell Fate by 3D Scaffold Structures through the Control of Cell Shape. Biomaterials, 32, 9188-9196. http://dx.doi.org/10.1016/j.biomaterials.2011.08.054

[20] Sahoo, S., Ang, L.T., Goh, J.C. and Toh, S.L. (2010) Growth Factor Delivery through Electrospun Nanofibers in Scaffolds for Tissue Engineering Applications. Journal of Biomedical Materials Research Part B: Applied Biomaterials, 93, 1539-1550.

[21] Zhang, X., Baughman, C.B. and Kaplan, D.L. (2008) In Vitro Evaluation of Electrospun Silk Fibroin Scaffolds for Vascular Cell Growth. Biomaterials, 29, 2217-2227. http://dx.doi.org/10.1016/j.biomaterials.2008.01.022

[22] Limbourg, A., Korff, T., Napp, L.C., Schaper, W., Drexler, H. and Limbourg, F.P. (2009) Evaluation of Postnatal Arteriogenesis and Angiogenesis in a Mouse Model of Hind-Limb Ischemia. Nature Protocols, 4, 1737-1748. http://dx.doi.org/10.1038/nprot.2009.185

[23] Cao, L. and Mooney, D.J. (2007) Spatiotemporal Control over Growth Factor Signaling for Therapeutic Neovascularization. Advanced Drug Delivery Reviews, 59, 1340-1350. http://dx.doi.org/10.1016/j.addr.2007.08.012

[24] Jakobsson, A. and Nilsson, G.E. (1993) Prediction of Sampling Depth and Photon Pathlength in Laser Doppler Flowmetry. Medical \& Biological Engineering \& Computing, 31, 301-307. http://dx.doi.org/10.1007/BF02458050

[25] Chalothorn, D., Clayton, J.A., Zhang, H., Pomp, D. and Faber, J.E. (2007) Collateral Density, Remodeling, and VEGF -An Expression Differ Widely between Mouse Strains. Physiological Genomics, 30, 179-191. http://dx.doi.org/10.1152/physiolgenomics.00047.2007

[26] Li, Y., Song, Y., Zhao, L., Gaidosh, G., Laties, A.M. and Wen, R. (2008) Direct Labeling and Visualization of Blood Vessels with Lipophilic Carbocyanine Dye DiI. Nature Protocols, 3, 1703-1708. http://dx.doi.org/10.1038/nprot.2008.172

[27] Hasan, M.R., Herz, J., Hermann, D.M. and Doeppner, T.R. (2012) Visualization of Macroscopic Cerebral Vessel Anatomy-A New and Reliable Technique in Mice. Journal of Neuroscience Methods, 204, 249-253. 
http://dx.doi.org/10.1016/j.jneumeth.2011.11.024

[28] Schmidt, C., Bezuidenhout, D., Beck, M., Van der Merwe, E., Zilla, P. and Davies, N. (2009) Rapid Three-Dimensional Quantification of VEGF-Induced Scaffold Neovascularisation by Microcomputed Tomography. Biomaterials, 30, 5959-5968. http://dx.doi.org/10.1016/j.biomaterials.2009.07.044 